\title{
La psicología clínica en las publicaciones hispanoamericanas: Un análisis bibliométrico del período 2012-2014
}

\section{Clinical psychology in hispanic american journals: A bibliometric analysis from 2012 to 2014}

\author{
Claudia Bregman \\ Fundación AIGLÉ, Argentina \\ Wilson López-López \\ Pontificia Universidad Javeriana, Colombia \\ Fernando García \\ Fundación AIGLÉ, Argentina
}

(Rec: 11 agosto 2015 / Acept: 17 octubre 2015)

\begin{abstract}
Resumen
Este estudio bibliométrico se realizó con el objeto de conocer el estado del arte en el campo de la psicología clínica en Hispanoamérica. Se analizaron 16 de un total de 18 revistas de la región que se encuentran indexadas en Scopus bajo el descriptor Psicología. Dos fueron excluidas de la muestra: una por no encontrarse en ella ningún artículo sobre psicología clínica y otra por haber sido discontinuada. Se procesaron los volúmenes correspondientes al período entre 2012 y 2014. Se clasificaron 769 artículos pertinentes a psicología clínica de un total de 1534. Los resultados mostraron la siguiente distribución: psicopatología (38\%), evaluación (24\%), promoción y prevención (17\%), psicoterapia (10\%), epidemiología (4\%), otras intervenciones (3\%), formación $(1 \%)$ y otros $(3 \%)$. Se concluye señalando fortalezas y debilidades de los resultados y las implicancias que éstos tienen en la práctica clínica.

Palabras clave: estudio bibliométrico, psicología clínica e Hispanoamérica.
\end{abstract}

\begin{abstract}
This bibliometric study was performed with the aim of surveying the state-of-the-art of clinical psychology in Hispanic America. Sixteen out of eighteen Hispanic America journals, indexed in Scopus under the Psychology descriptor, were analyzed. Two journals had to be discarded for different reasons: one of them did not have any articles about clinical psychology and the other had been discontinued. The volumes considered for the survey spanned a two-year period, from 2012 to 2014. There are a total of 1534 original articles, out of which 769 were related to clinical psychology. Results showed the following distribution: psychopathology (38\%), assessment (24\%), promotion and prevention (17\%), psychotherapy (10\%), epidemiology (4\%), others interventions $(3 \%)$, training $(1 \%)$, others $(3 \%)$. It concludes by pointing out strengths and weaknesses of the results and the implications that these have in clinical practice. Keywords: bibliometric study, clinical psychology, Hispanic America
\end{abstract}




\section{Introducción}

El desarrollo de la ciencia depende, en gran medida, de la posibilidad de debatir, contrastar y compartir las hipótesis, teorías y hallazgos que se van produciendo en el seno de cada campo específico. Por lo tanto, las disciplinas científicas requieren líneas efectivas de comunicación tanto en la generación de nuevas ideas como en la difusión de las mismas, ya sea en el avance de resultados de investigación como en el planteamiento de líneas a desarrollar.

Las revistas científicas constituyen un elemento privilegiado del sistema de comunicación entre profesionales e investigadores. Son unidades de análisis que permiten comprender cómo un área de conocimiento se encuentra organizada en determinado momento y a lo largo del tiempo. Los estudios bibliométricos, basados en los aspectos cuantificables de la literatura científica, vienen consolidándose desde hace unos años como uno de los principales instrumentos en el análisis objetivo de los sistemas científicos y del desarrollo de la ciencia.

El campo de la psicología clínica está orientado al estudio y cuidado de la salud mental y del comportamiento de los individuos y grupos, como así también dirigido a la promoción de la adaptación y del desarrollo personal. Se centra en el desarrollo intelectual, emocional, biológico, psicológico, social y en los aspectos conductuales del funcionamiento humano durante toda la vida, en diferentes culturas y en todos los niveles socioeconómicos (American Psychological Association, 2015).

Diferentes estudios han analizado el desarrollo de la producción de la psicología en español a través de indicadores bibliométricos (Olivas-Ávila, Musi-Lechuga, Quevedo-Blasco y Luna-Hernández, 2012; Rey-Anacona, Martínez-Gómez y Guerrero-Rodríguez, 2009; López-López, García-Cepero, Aguilar Bustamante, Silva y Aguado López, 2010; QuevedoBlasco y López-López, 2011). Otros consideraron para su análisis sólo revistas de la región latinoamericana (Riffo Allende, 2011; Vera-Villarroel, López-López, Lillo y Silva, 2011; Liberatore y Hermosilla, 2008, Quevedo-Blasco y López-López, 2010). Y también algunos analizaron específicamente temas vinculados con la psicología clínica (Vera-Villarroel y Mustaca, 2006; González, 2008; Agudelo, Bretón-López y Buela-Casal, 2003; Ariza y Granados, 2012; Lillo y Martini, 2013) .

Los aportes anteriores enfocaron diferentes aspectos del material publicado: diferentes países, distintos períodos y/o variados objetivos y metodologías. Esta propuesta es estudiar qué reflejan las 16 publicaciones científicas hispanoamericanas de psicología, indexadas en Scopus, sobre psicología clínica durante el período 2012-2014. Se realizó un estudio bibliométrico cuyo énfasis está puesto en las subdisciplinas de la psicología clínica y en el período más reciente.

\section{Método}

\section{Unidad de análisis}

Frente a la gran variedad de publicaciones sobre psicología, para el presente estudio descriptivo-retrospectivo se seleccionaron las publicaciones hispanoamericanas indizadas en la base de Scopus bajo el descriptor psicología. El período estudiado abarca del 2012 al 2014. En Scopus se encuentran bajo este rótulo 1.042 publicaciones, de las cuales se analizaron 16 de un total de 18 . Se excluyeron 2, una por estar discontinuada y otra por no contener artículos vinculados con la temática de este trabajo. Se incluyeron: Acta Colombiana de Psicología, Avances en Psicología Latinoamericana, Interdisciplinaria, International Journal of Psychological Research (Psykhe), Revista Argentina de Clínica Psicológica, Revista Colombiana de Psicología, Revista Iberoamericana de Diagnóstico y Evaluación Psicológica, Revista Latinoamericana de Psicología, Revista Mexicana de Análisis de la Conducta, Revista Mexicana de Neurociencia, Revista Mexicana de Psicología, Salud Mental, Suma Psicológica, Terapia Psicológica y Universitas Psychologica. En la Tabla 1 se describen en detalle estas publicaciones y se especifican las siglas con las cuales serán referidas en el artículo.

Los datos se obtuvieron a través de la web. En algunas revistas, el acceso a la información se realizó mediante su propio sitio y en otras se obtuvo de Redalyc.

Se seleccionaron solamente los trabajos originales y las revisiones publicadas en las revistas, durante el período anteriormente mencionado. Se computarizaron todos los artículos según los siguientes criterios:

1- Volumen de la producción (número de documentos) durante el período estudiado.

2- Tipos de autoría: autoría simple (número de documentos firmados por un solo autor) y autoría múltiple (número de documentos firmados por dos o más autores).

3- Porcentajes de la colaboración internacional (CI) y de colaboración nacional (CN). El número de documentos en CI son los que firmó con la colaboración de al menos un autor que tiene afiliación en una institución extranjera. 
Tabla 1. Descripción de las revistas indexadas en Scopus en el periodo 2012-2014

\begin{tabular}{|c|c|c|c|c|}
\hline Revistas & País & ISSN & Institución editora & Frecuencia \\
\hline $\begin{array}{l}\text { Revista Argentina de } \\
\text { Clínica Psicológica } \\
\text { (RACP) }\end{array}$ & Argentina & $\begin{array}{l}0327-6716 \\
1851-7951\end{array}$ & Fundación AIGLÉ & Cuatrimestral \\
\hline Interdisciplinaria (ID) & Argentina & $\begin{array}{l}0325-8203 \\
1668-7027\end{array}$ & $\begin{array}{l}\text { Centro Interamericano de } \\
\text { Investigaciones Psicológicas y } \\
\text { Ciencias Afines (CIIPCA) }\end{array}$ & Semestral \\
\hline $\begin{array}{l}\text { Revista Iberoamericana } \\
\text { de Diagnóstico y } \\
\text { Evaluación Psicológica } \\
\text { (RIDEP) }\end{array}$ & Argentina & $1135-3848$ & $\begin{array}{l}\text { Asociación Iberoamericana de } \\
\text { Diagnóstico y Evaluación } \\
\text { Psicológica }\end{array}$ & Semestral \\
\hline Terapia Psicológica (TP) & Chile & $\begin{array}{l}0716-6184 \\
0718-4808\end{array}$ & $\begin{array}{l}\text { Sociedad Chilena de Psicología } \\
\text { Clínica }\end{array}$ & Cuatrimestral \\
\hline Psykhe & Chile & $\begin{array}{l}0717-0297 \\
0718-2228\end{array}$ & $\begin{array}{l}\text { Pontificia Universidad Católica } \\
\text { de Chile }\end{array}$ & Semestral \\
\hline $\begin{array}{l}\text { Universitas Psychologica } \\
\text { (UP) }\end{array}$ & Colombia & $\begin{array}{l}1657-9267 \\
2011-2777\end{array}$ & Pontificia Universidad Javeriana & Cuatrimestral \\
\hline $\begin{array}{l}\text { Revista Latinoamericana } \\
\text { de Psicología (RLP) }\end{array}$ & Colombia & $0120-0534$ & $\begin{array}{l}\text { Fundación Universitaria Konrad } \\
\text { Lorenz }\end{array}$ & Cuatrimestral \\
\hline $\begin{array}{l}\text { Acta Colombiana de } \\
\text { Psicología (Acta) }\end{array}$ & Colombia & $\begin{array}{l}0123-9155 \\
1909-9811\end{array}$ & $\begin{array}{l}\text { Universidad Católica de } \\
\text { Colombia }\end{array}$ & Semestral \\
\hline $\begin{array}{l}\text { International Journal of } \\
\text { Psychological Research } \\
\text { (IJPR) }\end{array}$ & Colombia & $\begin{array}{l}2011-2084 \\
2011-7922\end{array}$ & Universidad San Buenaventura & Semestral \\
\hline $\begin{array}{l}\text { Revista Colombiana de } \\
\text { Psicología (RCP) }\end{array}$ & Colombia & $\begin{array}{l}0121-5469 \\
2344-8644\end{array}$ & $\begin{array}{l}\text { Universidad Nacional de } \\
\text { Colombia }\end{array}$ & Semestral \\
\hline $\begin{array}{l}\text { Avances en Psicología } \\
\text { Latinoamericana } \\
\text { (Avances) }\end{array}$ & Colombia & $\begin{array}{l}1794-4724 \\
2145-4515\end{array}$ & Universidad del Rosario & Cuatrimestral \\
\hline $\begin{array}{l}\text { Suma Psicológica } \\
\text { (Suma) }\end{array}$ & Colombia & $\begin{array}{l}0121-4381 \\
2145-9797\end{array}$ & $\begin{array}{l}\text { Fundación Universitaria Konrad } \\
\text { Lorenz }\end{array}$ & Semestral \\
\hline Salud Mental (SM) & México & $0185-3325$ & INPRFM & Bimestral \\
\hline $\begin{array}{l}\text { Revista Mexicana de } \\
\text { Análisis de la Conducta } \\
\text { (RMCP) }\end{array}$ & México & $\begin{array}{l}0185-4534 \\
2007-0802\end{array}$ & $\begin{array}{l}\text { Sociedad Mexicana de Análisis } \\
\text { de la Conducta }\end{array}$ & Cuatrimestral \\
\hline $\begin{array}{l}\text { Revista Mexicana de } \\
\text { Psicología (RMP) }\end{array}$ & México & 0185-6073 & $\begin{array}{l}\text { Sociedad Mexicana de } \\
\text { Psicología }\end{array}$ & Semestral \\
\hline $\begin{array}{l}\text { Revista Mexicana de } \\
\text { Neurociencia (RMN) }\end{array}$ & México & $1665-5044$ & $\begin{array}{l}\text { Academia Mexicana de } \\
\text { Neurología }\end{array}$ & Bimestral \\
\hline
\end{tabular}

Sólo los pertinentes a psicología clínica se clasificaron, en primer lugar teniendo en cuenta si correspondían o no a investigaciones empíricas y en segundo lugar considerando su temática. Para establecer las categorías se tuvieron en cuenta las subdisciplinas en las cuales se suele organizar la psicología clínica (Trull, 2013; Agudelo et al., 2003 y Rey et al., 2009), quedando establecidas: epidemiología, evaluación, psicoterapia, otras intervenciones, promoción y prevención, formación y otros.
Algunos de estos autores (Trull, 2013) incluyen psicología comunitaria y psicología forense. Sin embargo, estas subdisciplinas no se incluyeron debido a las particularidades que tienen y que las alejan bastante del consenso que existe en nuestra región sobre lo que los clínicos hacen en su actividad cotidiana (Montero, 2010).

La clasificación se realizó en forma excluyente. Es decir, en cuanto a su contenido, cada artículo fue clasificado en 
una sola categoría. En algunos casos un trabajo podría ser considerado en más de una, pero se lo incluyó en la categoría que, a criterio de los jueces, fuera la que mejor representaba su contenido. Para lograr aumentar la fiabilidad de la clasificación, todos los artículos fueron revisados por dos jueces. En los casos en que no hubo acuerdo entre ellos se acudió a un tercer juez para tomar la decisión.

Cabe aclarar que las clasificaciones no tienen valor de verdad sino que son más o menos relevantes para el conocimiento de una porción de la realidad. Como en toda clasificación, existen "mejores ejemplares" de cada taxón y otros que se encuentran más en la periferia. Para tener un panorama más claro respecto de los contenidos de las categorías antes mencionadas, se las volvió a dividir por temáticas. A pesar de eso, en algunos casos fue difícil clasificar algunos trabajos. Esto se resolvió agregando la categoría residual "Otros", que terminó albergando, finalmente, sólo una cantidad reducida de artículos.

Para ilustrar mejor el contenido de cada categoría se muestra el título de un artículo a modo de "prototipo". Esta concepción sigue los trabajos sobre principios de categorización desarrollados por Rosch y sus colaboradores (1975 y 1978). La elección de los artículos se realizó por consenso entre los autores, del mismo modo que la inclusión de los mismos en las categorías anteriormente descritas.

\section{Resultados}

El primer grupo de datos que se presentan tiene como objetivo describir la producción de artículos de acuerdo a: cantidad de documentos, distribución en las revistas, autoría y procedencia.

Los datos provienen de 16 revistas hispanoamericanas indizadas en la base de Scopus. Siete de ellas son editadas en Colombia (ver tabla 1).

Se han publicado en toda la región, durante el período 2012-2014, 46 números con un total de 1534 artículos, de los cuales 769 corresponden a psicología clínica, los que fueron objeto del presente estudio.

La Tabla 2 presenta el total de artículos originales de acuerdo a una primera categorización, según su pertenencia a la psicología clínica.

Los 769 artículos están firmados por 2.723 autores. En la Tabla 3 se puede observar la distribución del tipo de autoría en cada una de las publicaciones. Universitas Psychologica es la que más cantidad de artículos cuenta con la firma de
Tabla 2. Cantidad de artículos sobre psicología clínica publicados por cada revista en el período 2012-2014.

\begin{tabular}{lc}
\hline Revista & $\boldsymbol{N}^{\boldsymbol{o}}$ de artículos \\
\hline Acta (1) & 45 \\
Avances (2) & 53 \\
IJPR (3) & 41 \\
ID (4) & 18 \\
Psykhe (5) & 16 \\
RACP(6) & 75 \\
RCP (7) & 32 \\
RIDEP (8) & 39 \\
RLP (9) & 30 \\
RMAC (10) & 7 \\
RMN (11) & 24 \\
RMP (12) & 23 \\
SM (13) & 124 \\
Suma (14) & 16 \\
TP (15) & 78 \\
UP (16) & 148 \\
Total & $\mathbf{7 6 9}$ \\
\hline
\end{tabular}

(1) Acta Colombiana de Psicología, (2) Avances en Psicología Latinoamericana, (3) International Journal of Psychological Research, (4) Interdisciplinaria, (5) Psykhé, (6) Revista Argentina de Clínica Psicológica, (7) Revista Colombiana de Psicología, (8) Revista Iberoamericana de Diagnóstico y Evaluación Psicológica, (9) Revista Latinoamericana de Psicología, (10) Revista Mexicana de Análisis de la Conducta, (11) Revista Mexicana de Neurociencia, (12) Revista Mexicana de Psicología, (13) Salud Mental, (14) Suma Psicológica, (15) Terapia Psicológica y (16) Universitas Psychologica.

un solo autor. El $42 \%$ de los autores está concentrado en los trabajos producidos por 3 y 4 colegas. Salud Mental es la revista que tiene más artículos colectivos, ya que 2 los firman 15 autores, uno 16 y otro 18 .

Respecto al origen, 26 países son los que contribuyen con 692 manuscritos. La mayor cantidad los aporta México con 180 trabajos, contando con un total de 733 firmas, y le sigue España con 147 artículos en los que participan 468 autores. A su vez, las cuatro revistas mexicanas publican al menos el 50\% de los artículos provenientes de su país.

Con el objetivo de hacer una aproximación a las relaciones entre grupos de investigación de los países de la región entre sí y con países de otros continentes, se exploraron los trabajos elaborados en colaboración internacional. Éstos representan el $10 \%$, de los cuales 7 aportes son entre autores de países latinoamericanos, 38 entre colegas de España e 
Tabla 3. Distribución del índice de autoría por artículo, por revista, en el período 2012-2014.

\begin{tabular}{|c|c|c|c|c|c|c|c|c|c|c|c|c|c|c|c|c|c|}
\hline Autores & $\begin{array}{l}\text { Acta } \\
(1)\end{array}$ & $\begin{array}{c}\text { Avances } \\
\text { (2) }\end{array}$ & $\begin{array}{c}\text { IJPR } \\
(3)\end{array}$ & $\begin{array}{l}\text { ID } \\
\text { (4) }\end{array}$ & $\begin{array}{l}\text { Psykhe } \\
\text { (5) }\end{array}$ & $\begin{array}{c}\text { RACP } \\
\text { (6) }\end{array}$ & $\begin{array}{l}\mathrm{RCP} \\
(7)\end{array}$ & $\begin{array}{l}\text { RIDEP } \\
\text { (8) }\end{array}$ & $\begin{array}{l}\text { RLP } \\
(9)\end{array}$ & $\begin{array}{c}\text { RMAC } \\
(10)\end{array}$ & $\begin{array}{c}\text { RMN } \\
(11)\end{array}$ & $\begin{array}{l}\text { RMP } \\
(12)\end{array}$ & $\begin{array}{l}\text { SM } \\
(13)\end{array}$ & $\begin{array}{c}\text { Suma } \\
\text { (14) }\end{array}$ & $\begin{array}{c}\text { TP } \\
(15)\end{array}$ & $\begin{array}{c}\text { UP } \\
(16)\end{array}$ & Total \\
\hline 1 & 4 & 3 & 2 & 3 & 2 & 7 & 3 & 6 & 4 & & 5 & 2 & 7 & 4 & 4 & 11 & 67 \\
\hline 2 & 14 & 12 & 15 & 5 & 4 & 21 & 10 & 12 & 9 & 2 & 5 & 3 & 18 & 2 & 21 & 34 & 187 \\
\hline 3 & 13 & 20 & 7 & 9 & 2 & 17 & 10 & 11 & 9 & 2 & 4 & 5 & 26 & 6 & 25 & 45 & 211 \\
\hline 4 & 6 & 9 & 7 & & 4 & 13 & 2 & 6 & 3 & 2 & 1 & 11 & 26 & & 13 & 24 & 127 \\
\hline 5 & 4 & 4 & 4 & 1 & 2 & 9 & 1 & 3 & & & 4 & 1 & 16 & 2 & 6 & 18 & 75 \\
\hline 6 & 3 & 1 & 3 & & 1 & 5 & 1 & & 4 & 1 & 2 & 1 & 12 & & 3 & 7 & 44 \\
\hline 7 & 1 & 2 & 2 & & & 2 & 2 & 1 & 1 & & 1 & & 4 & & 3 & 6 & 25 \\
\hline 8 & & 1 & & & & & 3 & & & & & & 5 & 1 & 1 & 2 & 13 \\
\hline 9 & & 1 & 1 & & & & & & & & 1 & & 4 & 1 & 1 & & 9 \\
\hline 10 & & & & & & & & & & & 1 & & 1 & & & & 2 \\
\hline 11 & & & & & 1 & & & & & & & & 1 & & 1 & 1 & 4 \\
\hline 12 & & & & & & 1 & & & & & & & & & & & 1 \\
\hline 15 & & & & & & & & & & & & & 2 & & & & 2 \\
\hline 16 & & & & & & & & & & & & & 1 & & & & 1 \\
\hline 18 & & & & & & & & & & & & & 1 & & & & 1 \\
\hline Total & 45 & 53 & 41 & 18 & 16 & 75 & 32 & 39 & 30 & 7 & 24 & 23 & 124 & 16 & 78 & 148 & 769 \\
\hline
\end{tabular}

(1) Acta Colombiana de Psicología, (2) Avances en Psicología Latinoamericana, (3) International Journal of Psychological Research, (4) Interdisciplinaria, (5) Psykhe, (6) Revista Argentina de Clínica Psicológica, (7) Revista Colombiana de Psicología, (8) Revista Iberoamericana de Diagnóstico y Evaluación Psicológica, (9) Revista Latinoamericana de Psicología, (10) Revista Mexicana de Análisis de la Conducta, (11) Revista Mexicana de Neurociencia, (12) Revista Mexicana de Psicología, (13) Salud Mental, (14) Suma Psicológica, (15) Terapia Psicológica y (16) Universitas Psychologica.

Tabla 4. Distribución de cantidad de artículos y cantidad de autores según país de origen de la autoría en el período 2012-2014.

\begin{tabular}{lrr}
\hline País & $\mathbf{N}^{0}$ de artículos & $\mathbf{N}^{0}$ de autores \\
\hline Argentina & 59 & 149 \\
Brasil & 51 & 180 \\
Canadá & 1 & 2 \\
Chile & 96 & 363 \\
Colombia & 83 & 279 \\
Costa Rica & 2 & 4 \\
Cuba & 6 & 27 \\
España & 147 & 468 \\
Guatemala & 1 & 3 \\
México & 180 & 733 \\
Perú & 5 & 8 \\
Portugal & 24 & 72 \\
Puerto Rico & 1 & 4 \\
USA & 13 & 35 \\
Venezuela & 5 & 20 \\
Otros europeos & 11 & 37 \\
Otros países & 7 & 15 \\
2 ó + & 7 & 34 \\
hispanoamericanos & & 121 \\
España + & 38 & $\mathbf{2 7 2 3}$ \\
hispanoamericanos & 32 & \\
2 ó + otros & $\mathbf{7 6 9}$ & \\
\hline Total & & \\
\hline
\end{tabular}




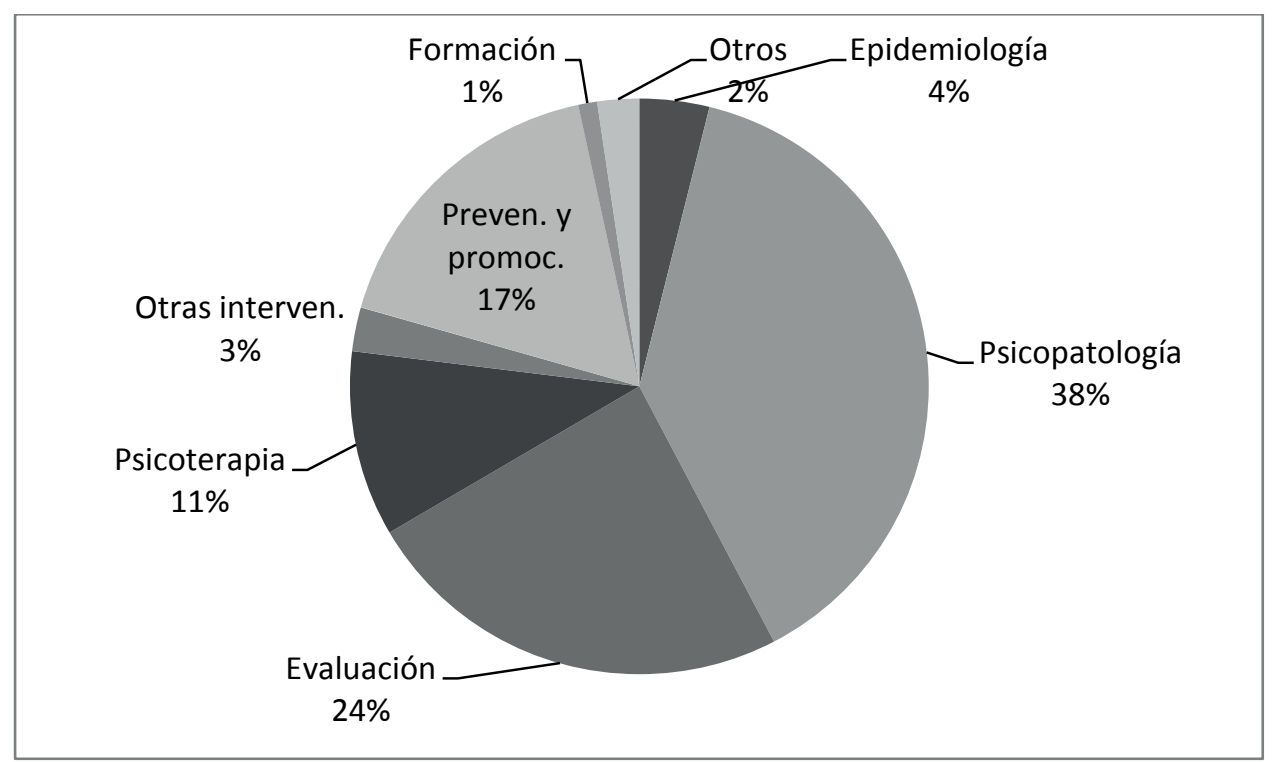

Figura 1. Distribución de artículos (\%) de temas de psicología clínica publicados en 16 revistas hispanoamericanas de idioma español, en el período 2012-2014.

Tabla 5. Distribución de cantidad de artículos de temas de psicología clínica en 16 revistas hispanoamericanas de idioma español, durante el periodo 2012-2014.

\begin{tabular}{|c|c|c|c|c|c|c|c|c|c|}
\hline Revista & Epidemiología & Psicopatología & Evaluación & Psicoterapia & $\begin{array}{c}\text { Otras } \\
\text { Interv. }\end{array}$ & Prom. y prev. & Formación & Otros & Totales \\
\hline Acta (1) & 3 & 13 & 11 & 4 & 1 & 12 & & 1 & 45 \\
\hline Avances (2) & & 24 & 12 & 5 & 2 & 9 & & 1 & 53 \\
\hline IJPR (3) & 2 & 22 & 9 & 2 & & 3 & & 3 & 41 \\
\hline ID (4) & & 4 & 7 & 2 & & 4 & & 1 & 18 \\
\hline Psykhe (5) & & 5 & 5 & 2 & & 2 & & 2 & 16 \\
\hline RACP (6) & 2 & 18 & 11 & 25 & 5 & 12 & & 2 & 75 \\
\hline $\mathrm{RCP}(7)$ & & 15 & 4 & 4 & & 8 & & 1 & 32 \\
\hline RIDEP (8) & 1 & 8 & 28 & & & 2 & & & 39 \\
\hline RLP (9) & 2 & 11 & 6 & 4 & & 6 & 1 & & 30 \\
\hline RMAC (10) & & 3 & & 2 & 2 & & & & 7 \\
\hline RMN (11) & 2 & 13 & 4 & 1 & 2 & 2 & & & 24 \\
\hline RMP (12) & 2 & 8 & 5 & 1 & & 7 & & & 23 \\
\hline SM (13) & 11 & 59 & 16 & 9 & 3 & 16 & 6 & 4 & 124 \\
\hline Suma (14) & & 6 & 5 & & 1 & 3 & & 1 & 16 \\
\hline ТP (15) & 1 & 23 & 22 & 10 & 1 & 18 & & 3 & 78 \\
\hline UP (16) & 4 & 63 & 42 & 9 & 2 & 24 & 1 & 3 & 148 \\
\hline Total & 30 & 295 & 187 & 80 & 19 & 128 & 8 & 22 & 769 \\
\hline
\end{tabular}

(1) Acta Colombiana de Psicología, (2) Avances en Psicología Latinoamericana, (3) International Journal of Psychological Research, (4) Interdisciplinaria, (5) Psykhe, (6) Revista Argentina de Clínica Psicológica, (7) Revista Colombiana de Psicología, (8) Revista Iberoamericana de Diagnóstico y Evaluación Psicológica, (9) Revista Latinoamericana de Psicología, (10) Revista Mexicana de Análisis de la Conducta, (11) Revista Mexicana de Neurociencia, (12) Revista Mexicana de Psicología, (13) Salud Mental, (14) Suma Psicológica, (15) Terapia Psicológica, (16) Universitas Psychologica. 


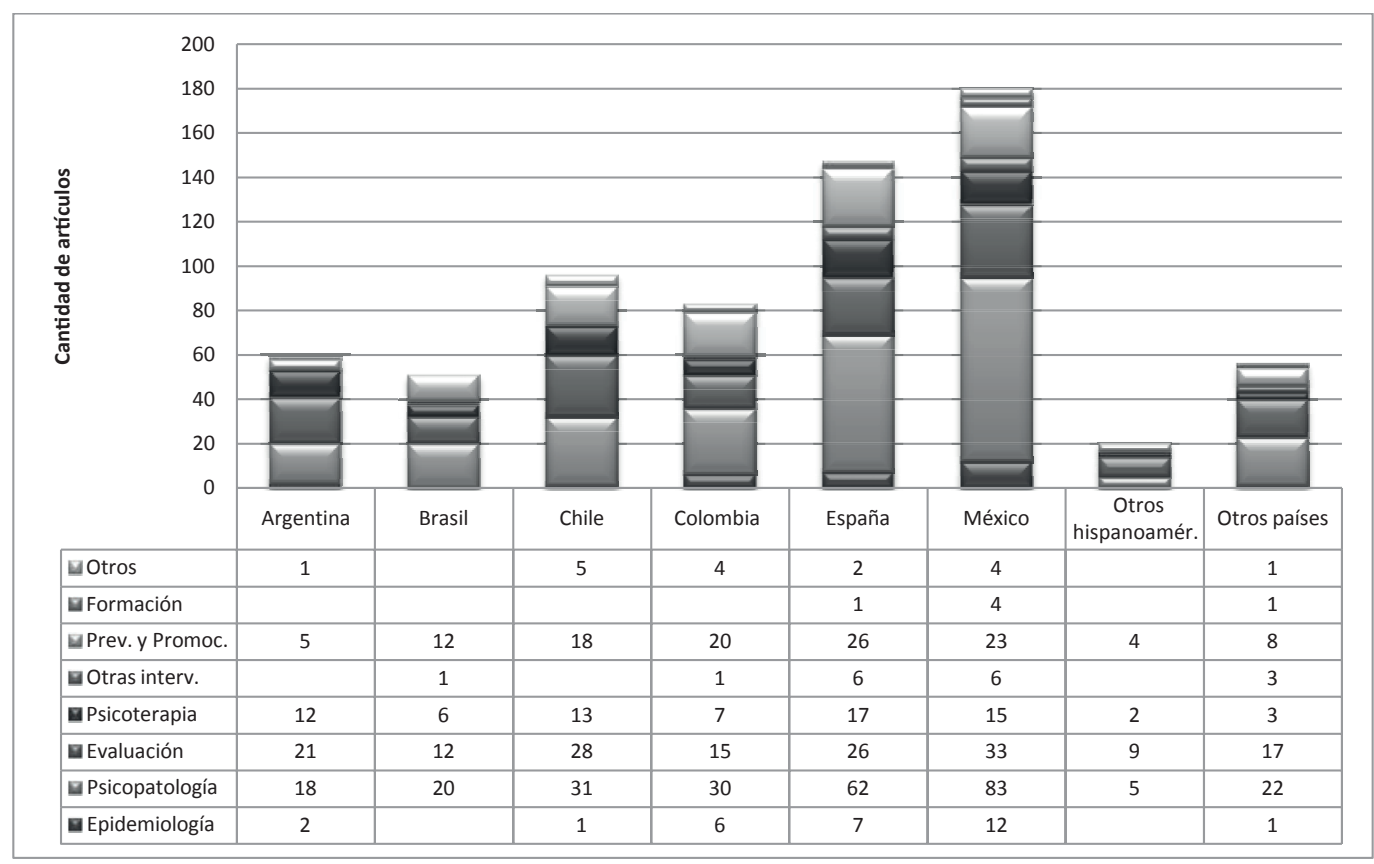

Figura 2. Temas de psicología clínica distribuidos por procedencia de autoría, en el período 2012-2014.

Hispanoamérica y, por último, 32 trabajos provienen de otro tipo de participación. Los trabajos en colaboración internacional se encuentran distribuidos principalmente entre Universitas Pychologica (31\%), Salud Mental (18\%) y Terapia Psicológica (14\%). Si se considera el origen de las publicaciones: Colombia publica el $55 \%$ de todos los trabajos de colaboración internacional, México el 21\%, Chile el 14\% y Argentina el 10\%.

A continuación se expondrá el conjunto de datos provenientes del análisis de los contenidos de los artículos.

En la Figura 1 se puede observar la distribución de los temas según las categorías antes explicitadas. El tema que congrega más cantidad de artículos es el referente a psicopatología (38\%), le sigue evaluación (24\%), promoción y prevención (17\%), psicoterapia (11\%), epidemiología (4\%), otras intervenciones (3\%), formación (1\%) y otros $(2 \%)$. A su vez, la Tabla 5 muestra la participación de los temas en las publicaciones estudiadas.

La Figura 2 expresa la distribución de los temas de psicología clínica según el país de procedencia.

En tanto, el estudio muestra que los trabajos basados en investigaciones empíricas son 651 y representando el $85 \%$. Por ende, el $15 \%$ de los trabajos corresponden a revisiones bibliográficas o teóricos. Se entiende por teórico a descripciones de constructos, intervenciones o reflexiones sin contrastación empírica. Este tipo de trabajos representa en psicoterapia el $30 \%$; en formación el $25 \%$; en otras intervenciones el $26 \%$; en psicopatología el $17 \%$, en salud-promoción y prevención el 14\%; en epidemiología el $10 \%$; en evaluación el $5 \%$; y en otros el $46 \%$.

\section{Epidemiología}

En esta categoría se encontraron 30 artículos, de los cuales 13 provienen de México, mientras que España aporta 7, Colombia 6, Argentina 2, y Chile y Malasia 1 cada uno. La distribución en las publicaciones, teniendo en cuenta el origen de la autoría, es la siguiente: 11 fueron publicados por SM, 4 por UP, 3 por Acta, 2 por IJPR, RACP, RLP, RMN, RMP y uno en RIDEP y TP.

La temática más difundida es la relacionada con las adicciones (23\%), como el trabajo de Villatoro, Medina-Mora, Fleiz Bautista, Moreno López, Oliva Robles et al. (2012) sobre los resultados de la encuesta nacional mejicana de población general. El otro tema que se reitera en 3 artículos es la depresión. En total, la población más estudiada es la de adolescentes y jóvenes adultos.

\section{Psicopatología}

Los 295 artículos pertenecientes a este taxón representan el $38 \%$ del total de lo publicado. Las revistas que más publican sobre este tema son UP, seguida de SM y luego Avances, TP, IJPR (ver Figura 3).

Si se los desagrega en función del origen de los autores, se observa que el $28 \%$ proviene de México, el $21 \%$ 


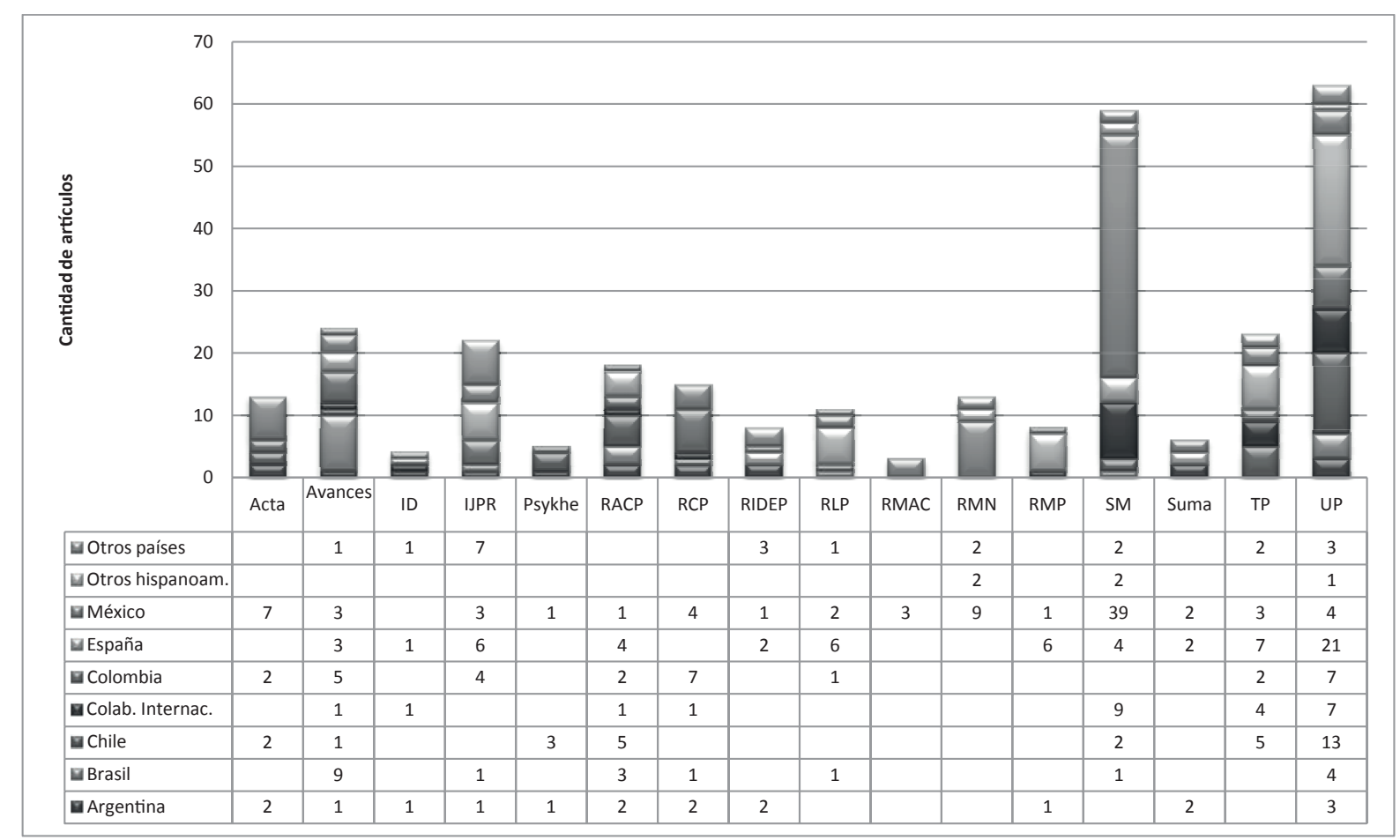

Figura 3. Distribución de artículos de psicopatología según revista de publicación y origen de autoría, en el período 2012-2014.

de España, el 11\% de Chile, el 10\% de Colombia, el 7\% de Brasil, el 6\% de Argentina, el 2\% de Estados Unidos, mientras que Portugal, Venezuela y Cuba aportan el 1\%, el $3 \%$ proviene de otros países y el $8 \%$ de la colaboración internacional. Si consideramos el porcentaje según el total de la producción, se advierte que el $46 \%$ del total proviene de México, seguido de España con el $42 \%$ de sus aportes, el 39\% de Brasil, el 36\% de Colombia, el 32\% de Chile, el $31 \%$ de Argentina, el $53 \%$ de los aportes de otros países y el $31 \%$ de la colaboración internacional.

$\mathrm{Al}$ reparar en el total de lo divulgado por cada revista surge que el $54 \%$ de IJPR es sobre este tópico al igual que la RMN, el 48\% de SM, el 47\% de RCP, el 45\% de Avances, UP y RMAC, el 43\%. En el resto de las publicaciones este tema representa menos del $40 \%$.

Las subcategorías detalladas a continuación se constituyeron de acuerdo al criterio de recurrencia de las temáticas, estableciéndose que el tópico debía estar presente al menos en 3 artículos.

Adicción: Se ubicaron 24 artículos, de los cuales 20 corresponden a abuso de sustancias, 3 al uso excesivo de internet y uno a la adicción al trabajo. Este último es el trabajo de Merino-Tejedor, Boada-Grau y PrizmicKuzmica (2014), en el cual se investiga la relación entre exceso de trabajo e irritación laboral, tanto cognitiva como emocional. El 50\% de los trabajos está dirigido al consumo de jóvenes y adolescentes, como el que indaga la relación entre asertividad y consumo de drogas (Velázquez Altamirano, Arellanez Hernández y Martínez García, 2012). Once de las contribuciones provienen de SM. Ocho son aportes de España y en dos de las cuatro contribuciones internacionales también participa el país ibérico sumando un total de 10; México colabora con 7, participando con otros países en otras 2 más.

Ansiedad: Se catalogaron en este rubro sólo 9 artículos, 2 de ellos centrados en el estudio de características del trastorno de ansiedad generalizada.

Antisocial: Se incorporaron 8 trabajos que se orientan al estudio del comportamiento y la personalidad antisocial desde diferentes niveles. Es así que el trabajo de Sánchez-Teruel y Robles-Bello (2013) estudia el modelo de personalidad de los "Big Five" aplicado al delincuente. Cuatro se orientan a los adolescentes.

Bipolar: De los 5 trabajos que ingresaron en esta categoría, 4 dirigen el foco al estudio de las familias y/o sus miembros como el elaborado por Santibáñez Fernández, Henson Maldonado y Troncoso Gallardo (2013). De estos aportes, 3 provienen de México y fueron publicados por $\mathrm{SM}$, los otros dos por RACP y provienen uno de Brasil y otro de Chile.

Burnout: Si bien este síndrome no se encuentra en ninguna de las clasificaciones internacionales (DSM-5 e ICD 10), 15 son los trabajos que lo exploran en diferentes ocupaciones: estudiantes de medicina, médicos oncólogos, 
enfermeros o profesionales de diferentes áreas, como el trabajo que presenta Franco-Bontempo (2014) sobre 243 profesionales de las distintas áreas de un centro de rehabilitación infantil. UP es la que publica 5 de estos artículos. Por otro lado, 5 provienen de México, de los cuales uno está escrito en colaboración con Colombia, país del cual provienen otros 2 más; dos son de Cuba, dos de España, que además comparte uno con Chile, que también presenta uno independiente como Brasil y Venezuela.

Déficit neurocognitivo: Los 7 trabajos que se incorporaron bajo este rubro corresponden al estudio de personas de edad avanzada, por ej. el trabajo de López-Higes, Prados, Montejo, Montenegro y Lozano (2014) sobre el deterioro cognitivo leve. Brasil, España y México aportan 2 cada uno y Venezuela uno. RMN y UP publican 3 cada una y Avances, uno.

Depresión: Este descriptor recibió una carga que representa el 9\% del total de psicopatología. Los 28 artículos referidos en este apartado abarcan los diferentes grupos etarios como el estudio desde diferentes niveles. Solo a modo de ejemplificar se señala el aporte de Gómez Maquet y Barrera Valencia (2012) sobre los aspectos neuropsicológicos asociados a síntomas depresivos en la infancia; y el de Ribeiro, dos Santos, Cazassa y da Silva Oliveira (2014), en el cual exploran, en una muestra de adultos, la relación entre los esquemas desadaptativos tempranos y los síntomas depresivos. El 50\% de estos artículos los publica SM, el 20\% UP y el resto se reparte entre otras publicaciones no alcanzando ninguna de ellas a más del $10 \%$. En cuanto al origen, 13 provienen de México, de los cuales dos están escritos en colaboración con Estados Unidos.

Estrés y trauma: En esta subcategoría ingresan 27 trabajos (9\%). El abanico temático abarca desde un estudio longitudinal, desde la infancia hasta la temprana adultez, para explorar cómo modulan las estrategias de afrontamiento a los acontecimientos vitales estresantes y los problemas de ajuste emocional (Estévez Campos, Oliva Delgado y Parra Jiménez, 2012), hasta cuál es el impacto que tiene en los periodistas cubrir la guerra contra el narcotráfico en México (Flores Morales, Reyes Pérez y Reidl Martínez, 2012). TP publica 7 de los artículos y 5 UP. Seis de los aportes son colaborativos.

Enfermedades somáticas: Bajo este descriptor se ubicaron 15 artículos, en los cuales el estudio se orienta a comprender aspectos psicopatológicos vinculados con enfermedades físicas, como el trabajo de Paredes y Kirchner Nebot (2012) sobre los síntomas psicológicos más allá de la ansiedad y depresión en pacientes con esclerosis múltiple. De México provienen 7 de los aportes y de España 6, de los cuales uno es una colaboración con Puerto Rico y Colombia.

Neurodesarrollo: Para este tema se registraron 31 colaboraciones, 3 referidas al autismo y 11 al trastorno por déficit de atención e hiperactividad. En este grupo hay contribuciones como las de Ocampo Gaviria y Sierra Fitzgerald (2013), orientadas al estudio de la compleja relación entre la memoria operativa y los trastornos de aprendizaje; y también los que demuestran la necesidad de estudios más precisos para caracterizar las variables afectadas en los trastornos por déficit de atención e hiperactividad (Puentes Rozo et al. 2014). Este es uno de los ítems que se encuentra más distribuido entre las publicaciones, igual que su origen.

Psicosis: Se agrupan en este rubro los 11 trabajos que exploran tanto los marcadores de vulnerabilidad como los aspectos de la personalidad en los trastornos psicóticos (Guízar Sánchez, Saracco Álvarez y Fresán Orellana, 2012). El 50\% está concentrado en SM. Tanto de España como de México provienen 3 aportes y uno es una colaboración entre ellos; otra es una colaboración entre México y Alemania; de Argentina provienen 2 y de Venezuela uno.

Alteraciones en el sueño: Bajo esta caracterización se registraron 3 artículos.

Problemas con la alimentación: Es uno de los taxones que más registros obtuvo con 24 originales. Se incluyeron tanto los factores de vulnerabilidad y riesgo como las alteraciones en la imagen corporal que presentan quienes tienen este tipo de perturbaciones y estuvieron orientados a diferentes grupos poblacionales como el trabajo de Toro-Alfonso, Urzúa y Sánchez Cardona (2012) con una muestra de hombres gay en Latinoamérica. Tres artículos publican cada una de las siguientes revistas: RACP, RCP, SM, TP y RLP, dos Acta y UP, uno Avances en Psicología Latinoamericana, IJPR, Psykhe, RMP y Suma. El 50\% proviene de México.

Autolesiones y suicidio: Dada la complejidad que presenta este tema, se decidió incluir en esta categoría aquellos trabajos en cuyo título figura una de las dos palabras con que se la definió. De los 14 estudios registrados, sólo tres no están orientados a la población adolescente. Fundamentalmente congregan la atención de los investigadores los aspectos de riesgo y predictores de la conducta suicida, siendo un ejemplo el trabajo de Guevara Werlang y Esteves Paranhos (2012). Seis artículos son 
publicados por SM, 3 por RACP, 2 por UP y RIDEP, uno por IJPR y otro por TP.

Violencia: En este grupo se clasificaron aquellos originales que versan sobre conductas intencionalmente dañinas como el abuso infantil, la agresión en la pareja, de padres a hijos o entre pares (bullying). treinta trabajos configuran este grupo. Varios se orientan a quienes son víctimas y otros, como el de Loinaz, Echeburúa y Ullate (2012), hacia la comprensión psicopatológica de los agresores. La concentración de aportes está en UP (50\%) y en RACP, RCP y TP (cada una 17\%). En cuanto al origen, principalmente está distribuido entre Chile, Colombia, España y México.

Personalidad: Es necesario aclarar que en esta categoría se concentraron aquellos estudios que indagan acerca del papel mediador de los rasgos y características de personalidad para la comprensión de los fenómenos psicopatológicos. Son 18 aportes. Un ejemplo es el trabajo presentado por Zanon y Hutz (2013) sobre la relación entre la disposición afectiva, los estilos de pensamiento, el neuroticismo y la satisfacción vital. Siete son publicados por UP y 4 por IJPR. El país que más aporta es Argentina.

\section{Evaluación}

Este grupo está conformado por 187 aportes, de los cuales 177 corresponden a pruebas psicométricas. Un primer acercamiento permite observar cómo es la distribución de este rubro en las publicaciones de acuerdo al origen del artículo (ver Figura 4). La mayor cantidad de artículos los publica UP, seguido de RIDEP.

Pero si se considera este rubro en relación al total de los aportes realizados por cada publicación, se observa que, como es de esperar por su especificidad, se destaca RIDEP contribuyendo con el $70 \%$ de su producción, seguido de ID (39\%), Psykhe y Suma (ambas 31\%), RMAC, TP y UP (28\% en las tres), Acta (24\%), Avances y RMP (ambas 23\%), IJPR (22\%), RLP (20\%), RMN (17\%), RACP (14\%), SM (13\%), RCP (12\%).

La procedencia de los trabajos se encuentran repartidos entre: México (18\%), Chile (15\%), España (14\%), Argentina (11\%), Colombia (8\%), Portugal (7\%), Brasil (6\%), Cuba (2\%), otros países (5\%) y colaboración internacional (14\%). Si se examina la producción relativa en función de la totalidad de cada país, se observa que el que más se centra en este tópico es Argentina (36\%), seguido de Chile (29\%), Brasil (23\%), Colombia, España y México (18\% cada uno), otros países $(12 \%)$ y colaboración entre países $(34 \%)$. Si bien la participación total de Portugal es de 24 trabajos, es para remarcar que el 50\% se encuentra en esta categoría y en su mayoría publicada por RIDEP.

En cuanto a los grupos etarios particulares, 30 están dirigidas a niños, 24 a adolescentes, 118 a adultos y 6 a adultos mayores. El $30 \%$ de los trabajos se realizaron con muestras de estudiantes. El uso de poblaciones similares

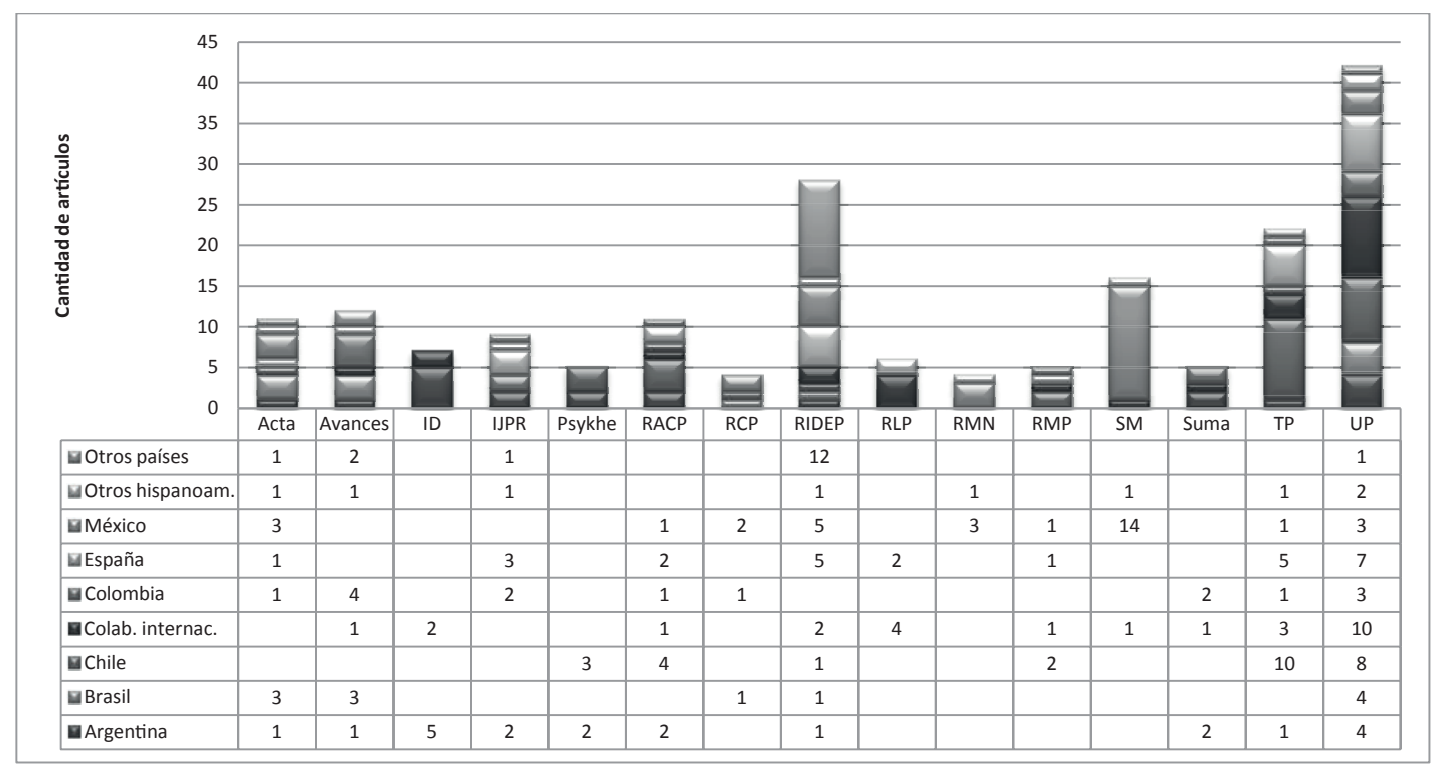

Figura 4. Distribución de artículos de evaluación según revista de publicación y origen de autoría, en el periodo 2012-2014. 


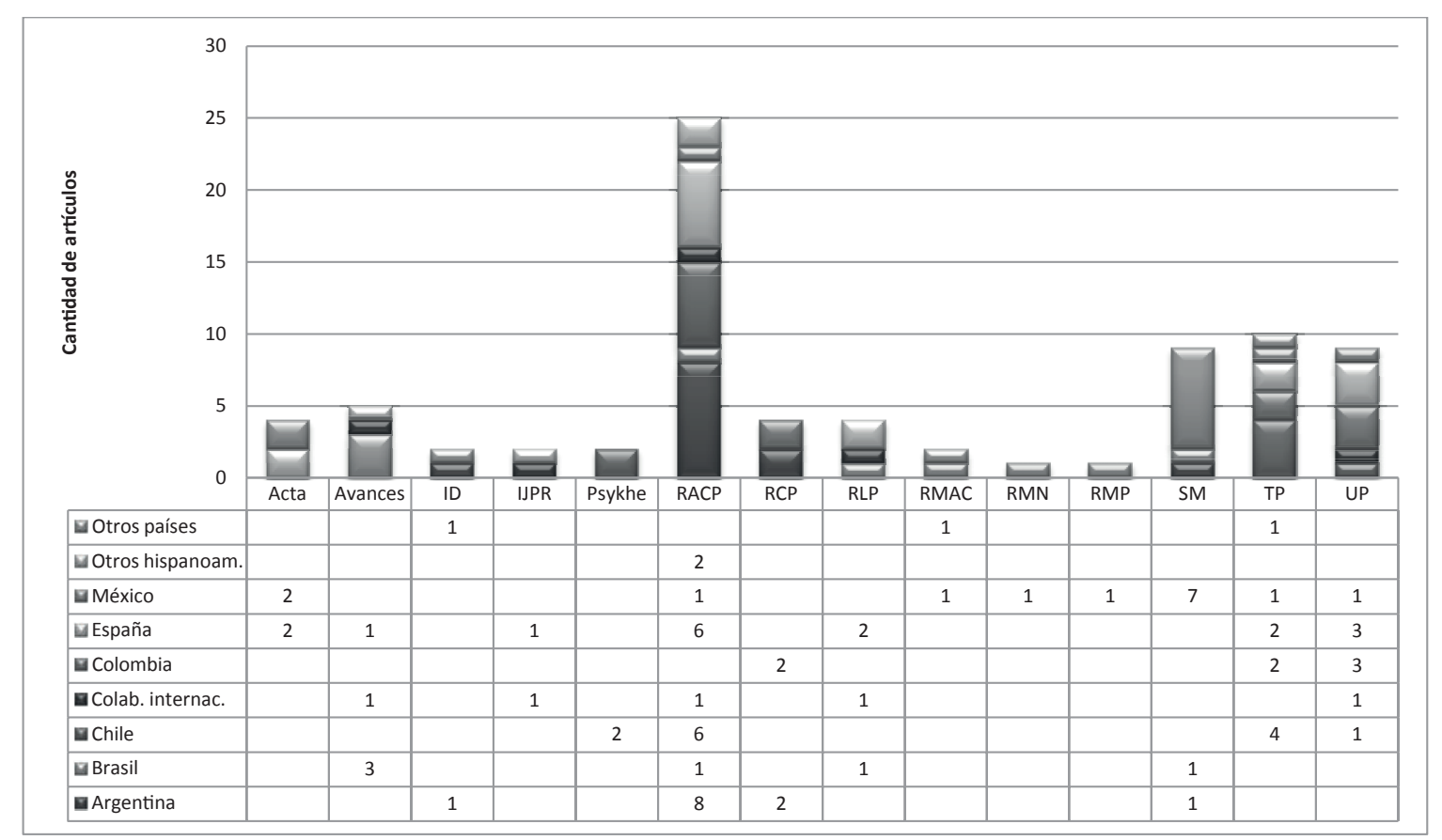

Figura 5. Distribución de artículos de psicoterapia según revista de publicación y origen de autoría, en el período 2012-2014.

presenta limitación, dado que no son representativas de toda la población, en especial en los estudios de validación de pruebas o listas de chequeo.

La gran mayoría de los trabajos son adaptaciones y validaciones de pruebas desarrolladas en otras regiones. Es para destacar que 24 (13\%) son propuestas originales.

En función de las variables que evalúan, se las subdividió en: adaptación y conducta (55\%), ansiedad, estrés y depresión (11\%), familia y pareja $(8 \%)$, inteligencia $(7 \%)$, neurocognitivos $(8 \%)$, personalidad $(11 \%)$, psicoterapia $(2 \%)$ para y otros. Sólo ilustrar los aportes en la construcción y validación de instrumentos citamos a García Meraz y Romero Palencia (2012), quienes se orientan a la pareja.

\section{Psicoterapia}

El 11\%, esto es 80 trabajos, corresponde a esta área. Si se atiende a la distribución según las publicaciones, RACP aporta el 31\%, TP el 13\%, UP, igual que SM, el 11\%, Avances el 6\%, Acta y RLP el 5\%, ID, IJPR, Psykhe, Revista Colombiana de Psicología y RMAC el 3\% (ver Figura 5).

En cuanto a la procedencia de los mismos: el $22 \%$ de España, el 19\% de México, el 16\% de Chile, el 15\% de Argentina, el 10\% de Colombia y el resto de otros países. Por otro lado, al atender esta categoría en relación al total de lo contribuido por cada publicación, surge que para Revista Argentina de Clínica Psicológica representa el 33\%, para
RMAC el 28\%, para RCP, RLP, TP y SM el 13\%, para Psykhe el 12\%, para ID el 11\%, para Acta y Avances el 9\%, para IJPR el 5\%, para UP, el 6\% y para RMN y RMP el 4\%. El $20 \%$ de los trabajos basaron su investigación en el estudio de caso único, tanto en diseños experimentales y observacionales como en presentación de casos (Roussos, 2007).

El criterio de análisis fue el tipo de investigación, quedando definidos los grupos según sean de resultados, procesos o variables que intervienen. Se registraron 30 aportes en el primero, 10 en el segundo y en el tercero 33, quedando conformada una cuarta categoría, otros, compuesta por 7 trabajos.

Al reparar en las temáticas, en las investigaciones de resultados se observa un grupo de estudios orientados a la evaluación de dispositivos y técnicas específicas, como el propuesto por Gómez, Bernaldo-de Quirós, Labrador, Fernández-Arias y Estupiñá (2014) sobre intervenciones psicoeducativas en dispositivo grupal.

Por otro lado, dentro de los estudios de procesos cobran particular relevancia las investigaciones sobre microprocesos de la comunicación verbal. El programa de investigación que lleva adelante el grupo chileno sobre los episodios de cambio y estancamiento en psicoterapia, es un buen representante (Fernández et al. 2012). Por su parte, en los estudios centrados en variables intervinientes se enfatiza en la variable del terapeuta (Moncada, Guerra Vio, Concha y Carvajal, 2014). 


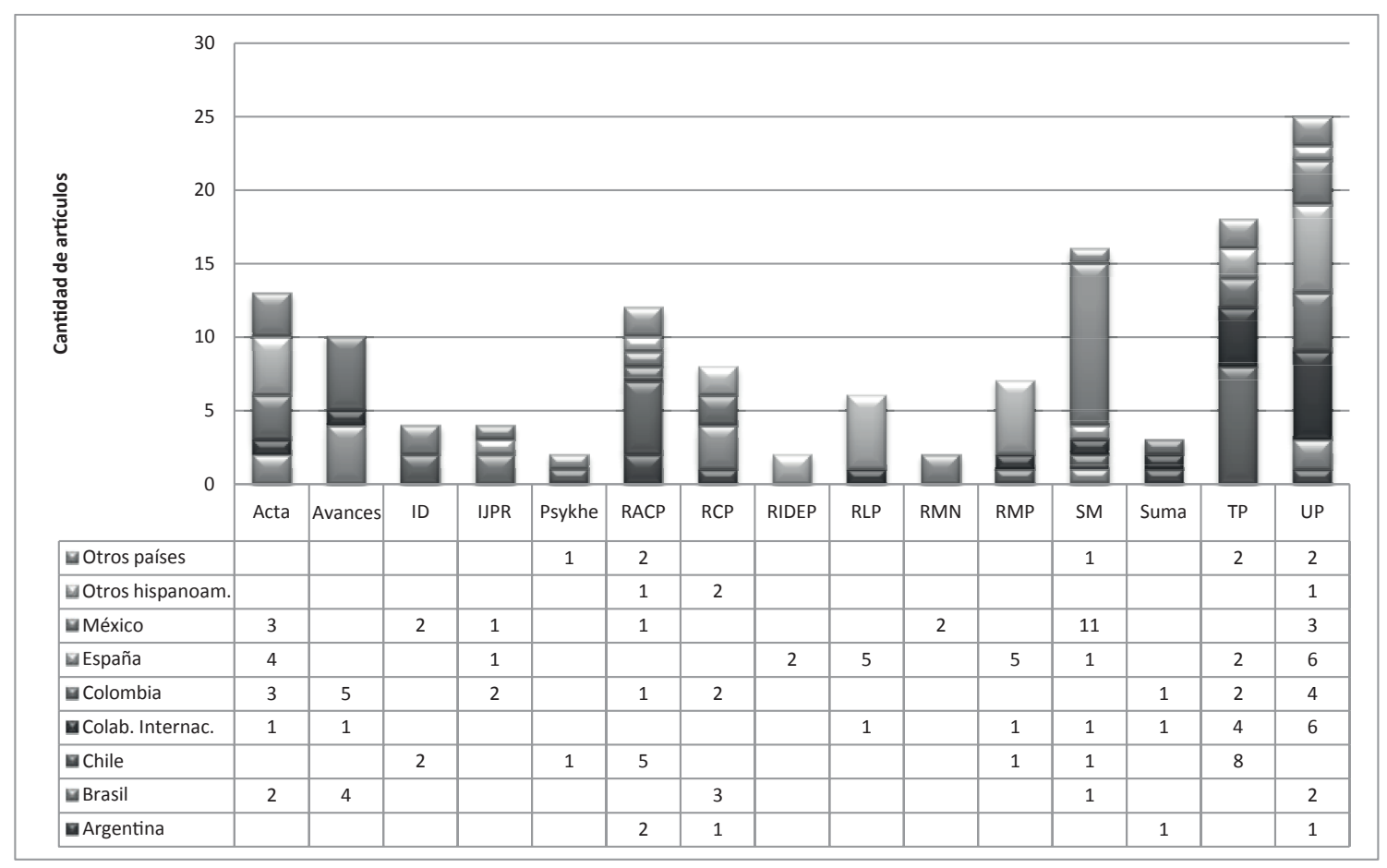

Figura 6. Distribución de artículos de salud-prevención y promoción según revista de publicación y origen de autoría, en el período 2012-2014.

\section{Otras intervenciones}

A este grupo pertenecen 19 trabajos que incluyen, entre otros, grupos de autoayuda, actividad física o el uso de la computadora para promover la independencia de niños con trastornos del espectro autista (Plavnick et al. 2014). México y España aportan 6 artículos cada uno, Estados Unidos 3, Colombia y Cuba uno cada uno, y 2 son producto de colaboración. RACP publica 5, SM 3, tanto Avances como RMAC, RMN y UP, 2 y Acta, Suma y TP 1 cada una.

\section{Salud-promoción y prevención}

Se computaron 132 manuscritos entre los cuales se encuentra un amplio abanico de temáticas: un modelo de prevención de discapacidad en niños con daño neurológico perinatal (Muñoz Ledo Rábago et al. 2013); y también el análisis que presenta Neves et al. (2012) en relación a las políticas en salud mental en Brasil.

El 24\% de los artículos provenientes de Brasil corresponden a este taxón, el 22\% de Colombia, el 19\% de Chile, el 18\% de España, el 12\% de México, el 8\% de Argentina, el 18\% de otros países y el 19\% de la colaboración internacional.

Al considerar la distribución de esta categoría según la publicación, se observa que el 19\% corresponde a UP, le sucede TP con el 14\%, seguida de SM con el 13\%, luego Acta y RACP cada una con el 9\%, Avances con el 7\%, RCP con el 6\%, aportando el 5\% tanto RLP como RMP, y el resto se reparte entre ID, Psykhe, RIDEP, RMN y Suma (ver Figura 6).

De su producción total, RMP destina el $30 \%$ a este rubro, Acta, el 27\%, RCP, el 25\%, TP, el 23\%, ID, el 22\% y RLP, el 20\%. El porcentaje de las demás es menor al 20\%.

\section{Formación}

Esta sección contiene sólo 8 aportes, de los cuales 6 son publicados por SM, 1 por RLP y otro por UP. Este último es un aporte italiano de Langher, Brancadoro, D'Angeli y Caputo (2014), orientado a explorar las representaciones de los estudiantes de psicología en relación a la residencia hospitalaria.

\section{Otros}

Compuestos por 18 trabajos que por su tema no corresponden a ninguna de las categorías descritas. Entre éstos se encuentran reflexiones sobre modelos teóricos, aspectos históricos, metodológicos y epistemológicos y estudios sobre publicaciones. 


\section{Conclusiones}

Este estudio pretende ofrecer un panorama acerca del cauce de la investigación en las publicaciones científicas sobre la psicología clínica, las áreas y temáticas de interés.

Por un lado permite vislumbrar ciertas características respecto a la circulación de la información. Los autores de habla hispana son los que más publican en estas revistas. El caudal más importante proviene de países de la región (México, Colombia, Chile y Argentina). Es muy significativa la contribución de autores de España, no sólo por la cantidad de contribuciones sino por la dispersión de sus artículos en diferentes publicaciones hispanoamericanas. En mucha menor medida se reciben aportes de otros países de fuera de la región, permitiendo estar representados, en un muy bajo porcentaje, todos los continentes. Se observa escasa presencia de trabajos de colaboración internacional $(10 \%)$. De estos trabajos, el porcentaje mayor se encuentra en los referidos a evaluación (34\%), psicopatología (31\%) y prevención y promoción (19\%).

Si bien estos datos no alcanzan para deducir el tipo de redes vigentes en el micro mundo de la investigación científica en psicología clínica, esbozan ciertas pautas acerca de la baja inclusión que tienen los aportes de la región en el entramado internacional (Quevedo-Blasco y López-López, 2010). Para tener una perspectiva más completa respecto de las características de la red de investigaciones hispanoamericanas sería necesario completarla con otros indicadores, tales como el estudio de citaciones en estas publicaciones y en las correspondientes a otras regiones (Chinchilla-Rodríguez, Miguel y de Moya-Anegón, 2015; Lillo y Martini, 2013).

Por otro lado se encuentra el análisis de la producción. $\mathrm{Al}$ analizar la distribución de las respuestas por áreas se infiere que la actividad de los investigadores está dirigida prioritariamente al estudio y evaluación de los fenómenos psicopatológicos en sus diferentes niveles de complejidad. Si se consideran en una visión global los estudios epidemiológicos, los aportes en el área de psicopatología y las técnicas orientadas a la evaluación de trastornos específicos, este conjunto conforma el $45 \%$ del total de las respuestas. Este es un claro indicador de la tendencia del campo en estudio, que coincide con investigaciones anteriores (Lillo y Martini, 2013; Airza y Granados, 2012; Riffo Allende, 2011; ReyAnacona, Martínez-Gómez y Guerrero-Rodríguez, 2009).

En relación a la temática, es para destacar la presencia de contribuciones respecto a perturbaciones en las que están comprometidos directamente los factores de riesgo sociales. En este trabajo se expresan en las categorías: violencia y burnout. Si se incluye dentro de este abanico a la correspondiente a adicciones y antisocial, este tópico configura el $25 \%$ del total de las respuestas al área de psicopatología. Por otro lado, entre los cuadros psicopatológicos, la depresión, estudiada en los diferentes grupos etarios, junto con los trastornos del neurodesarrollo en la población clínica infantil, son los que congregan más informes. Se encontró también una presencia significativa de problemáticas vinculadas con la alimentación, en concordancia con los estudios de evaluación para este ítem. El tema del suicidio se lo consideró con un taxón diferenciado de los otros y sus estudios se encuentran principalmente direccionados a los jóvenes.

Tanto en la categoría de evaluación como en la de prevención y promoción se manifiesta un interés por el rol de la familia y las redes sociales como factor protector dentro del campo de la salud mental.

Los desarrollos más limitados, comparados con el total de lo presentado, son el rubro psicoterapia y el correspondiente a otras intervenciones, datos que difieren si se los compara con estudios que incluyen en su análisis las revistas editadas en Iberoamérica (Lillo y Martini, 2013; Rey Anacona et al. 2009). Por los datos pareciera que la investigación en psicoterapia y en cómo intervenir para producir procesos de cambio en los padecimientos psicológicos sigue siendo un terreno aún no suficientemente explorado en la región, lo cual no contradice la presencia de grupos que dan cuenta de desarrollos que muestran tecnologías avanzadas.

Este análisis del corte transversal de las publicaciones científicas intenta ser una contribución acerca de las fortalezas y también debilidades en la disciplina.

Entre las fortalezas se podría mencionar que la temática con más artículos publicados es psicopatología con el $38 \%$ de trabajos. Esta área disciplinar de la clínica es su mayor aspecto de ciencia aplicada y es uno de los componentes que sustentan a los distintos programas de intervención. Otra fortaleza es que en segundo lugar aparecen los trabajos sobre evaluación psicológica -24\%-, lo que se alinea con la tradición de la psicología clínica desde su fundación. Además, esto complementa en muchos casos la investigación en psicopatología.

En tercer lugar aparecen los trabajos sobre prevención y promoción de la salud -17\%- .

Dejamos para el final lo que, a nuestro juicio, podría considerarse una debilidad: un $11 \%$ de los trabajos son sobre psicoterapia. Esto supone que la subdisciplina que suele ser considerada algo así como la raison d'être de los psicólogos clínicos, reúne una modesta cantidad de publicaciones comparadas con la totalidad. Esto cobra gran importancia de cara a la necesidad de la investigación y diseminación del conocimiento que permita que nuestras intervenciones estén cada vez más basadas en la evidencia en todas sus formas. 


\section{Referencias}

Agudelo, D., Bretón-López, J. y Buela-Casal, G. (2003). Análisis bibliométrico de las revistas de Psicología Clínica editadas en castellano. Psicothema, 15, 507-516.

American Psychological Association (2015). Clinical Psychology. Recuperado desde http://www.apa.org/ed/graduate/specialize/clinical.aspx

Ariza, T. y Granados, M. R. (2012). Análisis bibliométrico de las revistas iberoamericanas más relevantes afines a la Psicología Clínica y Salud del Journal CitationReport (2011). Terapia Psicológica, 30, 89-102.

Chinchilla-Rodríguez, Z., Miguel, S. y de Moya-Anegón, F. (2015). What factors affect the visibility of Argentinean publications in humanities and social sciences in Scopus? Some evidence beyond the geographic realm of research. Scientometrics, 102, 789-810.

Estévez Campos, R. M., Oliva Delgado, A. y Parra Jiménez, A. (2012). Acontecimientos vitales estresantes, estilo de afrontamiento y ajuste adolescente: un análisis longitudinal de los efectos de moderación. Revista Latinoamericana de Psicología, 44, 39-53.

Fernández, O., Herrera, P., Krause, M., Pérez, J. C., Valdés, N., Vilches, O. y Tomicic, A. (2012). Episodios de Cambio y Estancamiento en Psicoterapia: Características de la comunicación verbal entre pacientes y terapeutas. Terapia Psicológica, 30, 5-22.

Flores Morales, R., Reyes Pérez, V., Reidl Martínez, M. L. (2012). Síntomas de estrés postraumático (EPT) en periodistas mexicanos que cubren la guerra contra el narcotráfico. Suma Psicológica, 19, 7-17.

Franco-Bontempo, M.X. (2014). El síndrome de burnout en un centro de rehabilitación infantil de México. Revista Mexicana de Neurociencia, $15,74-80$.

García Meraz, M. y Romero Palencia, A. (2012). Mantenimiento en la relación de pareja: construcción y validación de dos escalas. Revista Iberoamericana de Diagnóstico y Evaluación Psicológica, 34, 133-155.

Gómez, L., Bernaldo-de Quirós, M., Labrador, F. J., Fernández-Arias, I., Estupiñá, F. J. y Blanco, C. (2014). Uso de técnicas de exposición en una clínica de psicología. Universitas Psychologica, 13, 715-724.

Gómez Maquet, Y. y Barrera Valencia, M. (2012). Aspectos neuropsicológicos asociados a la presencia de síntomas depresivos en niños escolarizados. Acta Colombiana de Psicología, 15, 111-118.

González, E. N. (2008). Análisis Cuantitativo del Primer Quinquenio de la Revista Argentina de Clínica Psicológica: 1992-1996. Revista Argentina de Clínica Psicológica, XVII, 277-282.

Guevara Werlang, B.S. y Esteves Paranhos, M. (2012). Identificación de Potencial Suicida en Adolescentes de 13 a 19 Años. Revista Argentina de Clínica Psicológica, XXI, 281-289.

Guízar Sánchez, D. P., Saracco Álvarez, R. y Fresán Orellana, A. (2012). Rasgos de personalidad en pacientes con esquizofrenia. Salud Mental, 35, 339-344.

Langher, V., Brancadoro, B., D’Angeli, M. y Caputo, A. (2014). Imagining future internship in profesional psychology: A study on university students' representations. Universitas Psychologica, 13, 1589-1601.

Liberatore, G. y Hermosilla, A. (2008). La Producción Científica Argentina en Psicología: Un Análisis de la Visibilidad e Impacto en el Ámbito Internacional y su Comparación con Países de la Región. Revista Interamericana de Psicología, 42, 507-512.

Lillo, S. y Martini, N. (2013). Principales Tendencias Iberoamericanas en Psicología Clínica. Un Estudio Basado en la Evidencia Científica. Terapia Psicológica, 31 (3), 363-371.

Loinaz, I., Echeburúa, E. y Ullate, M. (2012). Estilo de apego, empatía y autoestima en agresores de pareja. Terapia Psicológica, 30, 61-70.

López-López, W., García-Cepero, M.C., Aguilar Bustamante, M.C., Silva, L.M. y Aguado López, E. (2010). Panorama general de la producción académica en la psicología iberoamericana, 2005-2007. Papeles del Psicólogo, 31, 296-309.

López-Higes, R., Prados, J. M., Montejo, P., Montenegro, M. y Lozano, M. (2014). Is there a grammatical comprehension deficit in multidomain mild cognitive impairment? Universitas Psychologica,13,1569-1579.
Merino-Tejedor, E., Boada-Grau, J. y Prizmic-Kuzmaica, J. A. (2014). La relación entre la irritación laboral y la adicción al trabajo en una muestra española multiocupacional. Universitas Psychologica, 13, 477-489.

Moncada, L., Guerra Vio, C., Concha, F. y Carvajal, C. (2014). ¿Qué Revelan una Década Después los Estudios Chilenos sobre la Variable del Terapeuta? Un análisis bibliográfico. Revista Argentina de Clínica Psicológica, XXIII, 71-80.

Montero, M. (2010). Crítica, autocrítica y construcción de teoría en la psicología social latinoamericana. Revista Colombiana de Psicología, 19, 177-191.

Muñoz Ledo Rábago, P., Méndez Ramírez, I., Sánchez Pérez, C., Mandujano, M. y Valdés y Murata, Ch. (2013). Interacciones tempranas madre-niño y predicción de desarrollo motor mediante ecuaciones estructurales: Aplicación del modelo en niños con riesgo de daño neurológico perinatal. Interdisciplinaria, 30, 119-138.

Neves, R., Dimenstein, M., Paulon, S., Nardi, H., Bravo, O., De Medeiros Galvão, V., De Sousa Severo, A. K. y Figueiró, R. L. (2012). A saúde mental no sistema único de saúde do Brasil: duas realidades emanálise. Avances en Psicología Latinoamericana, 30, 356-368.

Ocampo Gaviria, T. y Sierra Fitzgerald, O. (2014). Análisis del funcionamiento de la memoria operativa en niños con trastornos en el aprendizaje. Acta Colombiana de Psicología, 17 (2), 81-90.

Olivas-Ávila, J. A., Musi-Lechuga, B., Quevedo-Blasco, R. y Luna Hernández, J. R. (2012). Índice de Internacionalidad de las revistas iberoamericanas de Psicología en el Journal Citation Report (2011). Revista Latinoamericana de Psicología, 44, 175-183.

Paredes, L. y Kirchner Nebot, S. (2012). Síntomas psicológicos en españoles enfermos de esclerosis múltiple: rol del género y relación con los años de diagnóstico. Revista Mexicana de Psicología, 29, 147-156.

Plavnick, J. B., Mariage, T., Englert, C. S., Constantine, K., Morin, L. y Skibbe, L. (2014). Promoting Independence During Computer Assisted Reading Instruction for Children with Autism Spectrum Disorders. Revista Mexicana de Modificación de la Conducta, 40, 85-105.

Puentes Rozo, P., Jiménez Figueroa, G., Pineda Alhucema, W., Pimienta Montoya, D., Acosta López, J., Cervantes Henríquez, M.L., Núñez Barragán, M. y Sánchez Rojas, M. (2014). Déficit en habilidades sociales en niños con trastorno por déficit de atención-hiperactividad, evaluados con la Escala BASC. Revista Colombiana de Psicología, 23, 95-106.

Quevedo-Blasco, R. y López-López, W. (2011). Situación de las revistas iberoamericanas de Psicología en el Journal Citation Report de 2010. Universitas Psychologica 10, 937-947.

Quevedo-Blasco, R. y López-López, W. (2010). Análisis bibliométrico de las revistas multidisciplinares de psicología recientemente incorporadas en la Web of Science (2008-2009). Psicologia: Reflexão e Crítica, 23, 384-408.

Rey-Anacona, C.A., Martínez-Gómez, J. A. y Guerrero-Rodríguez, S. I. (2009). Tendencias de los Artículos en Psicología Clínica en Iberoamérica. Terapia Psicológica, 27, 61-71.

Ribeiro, F., dos Santos, P. L., Cazassa, M. J. y da Silva Oliveira, M. (2014). Esquemas desadaptativos tempranos y síntomas depresivos: Estudio de comparación intergrupal. Revista Argentina de Clínica Psicológica, XXIII, 15-22.

Riffo Allende, G. (2011). Tendencia de los artículos de psicología clínica en Chile, Latinoamérica y España. Salud y Sociedad, 2, 90-99.

Rosch, E. (1975). Cognitive references points. Cognitive Psychology, 7, 532-547.

Rosch, E. (1978). Principles of categorization. En Rosch y Lloid (Ed). Cognition and Categorization. Hillsdale, N. J.: Lawrence Erlbaum Associates.

Roussos, A. J. (2007). El diseño de caso único en investigación en psicología clínica. Un vínculo entre la investigación y la práctica clínica. Revista Argentina de Clínica Psicológica, XVI, 261-269.

Sánchez-Teruel, D. y Robles-Bello, M.A. (2013). El modelo "Big Five" de personalidad y conducta delictiva. International Journal of Psychological Research, 6, 102-109.

Santibáñez Fernández, P., Henson Maldonado, I. y Troncoso Gallardo, E. (2013). Utilización del Family Function Style Scale en familias con un miembro diagnosticado con Trastorno Bipolar: Una mirada desde 
el paciente y la familia. Revista Argentina de Clínica Psicológica, XXII, 279-286.

Toro-Alfonso, J., Urzúa, M. A. y Sánchez Cardona, I. (2012). Actualización en Trastornos alimentarios. El Cuerpo del Delito: La imagen corporal e indicadores de trastornos alimentarios en una muestra de hombres gay de diez países latinoamericanos. Revista Argentina de Clínica Psicológica, XXI, 101-110.

Trull, T. y Prinstein M. J. (2013). Clinical Psychology. 8th Edition. Belmont: Wadsworth, Cengage Learning.

Velázquez Altamirano, M., Arellanez Hernández, J. L. y Martínez García, A. L. (2012). Asertividad y consumo de drogas en estudiantes mexicanos. Acta Colombiana de Psicología, 15, 131-141.

Vera-Villarroel, P. y Mustaca, A. (2006) Investigaciones en psicología clínica basadas en la evidencia en Chile y Argentina. Revista Latinoamericana de Psicología, 38, 551-556.

Vera-Villarroel, P., López-López, W., Lillo, S. y Silva, L.M. (2011). La producción científica en psicología latinoamericana: Un análisis de la investigación por países. Revista Latinoamericana de Psicología, 43, 95-104.

Villatoro, J., Medina-Mora, M. E., Fleiz Bautista, C., Moreno López, M., Oliva Robles, N., Bustos Gamiño, M., Fregoso Ito, D., Gutiérrez López, M.L. y Amador Buenabad, N. (2012). El consumo de drogas en México: Resultados de la Encuesta Nacional de Adicciones, 2011. Salud Mental, 35, 447-457.

Zanon, C. y Hutz, C. S. (2013). Affective disposition, thinking styles, neuroticism and life satisfaction. Universitas Psychologica, 12, 403-411. 
